\title{
Hybrid Digital-Analog Joint Source-Channel Coding for Broadcasting Correlated Gaussian Sources*
}

\author{
Hamid Behroozi, Fady Alajaji and Tamás Linder \\ Department of Mathematics and Statistics, Queen's University, Kingston, Ontario, Canada, K7L 3N6 \\ Email: \{behroozi, fady, linder\}@mast.queensu.ca
}

\begin{abstract}
We consider the transmission of a bivariate Gaussian source $S=\left(S_{1}, S_{2}\right)$ across a power-limited two-user Gaussian broadcast channel. User $i(i=1,2)$ observes the transmitted signal corrupted by Gaussian noise with power $\sigma_{i}^{2}$ and wants to estimate $S_{i}$. We study hybrid digital-analog (HDA) joint source-channel coding schemes and analyze these schemes to obtain achievable (squared-error) distortion regions. Two cases are considered: 1) source and channel bandwidths are equal, 2) broadcasting with bandwidth compression. We adapt HDA schemes of Wilson et al. [1] and Prabhakaran et al. [2] to provide various achievable distortion regions for both cases. Using numerical examples, we demonstrate that for bandwidth compression, a three-layered coding scheme consisting of analog, superposition, and Costa coding performs well compared to the other provided HDA schemes. In the case of matched bandwidth, a three-layered coding scheme with an analog layer and two layers, each consisting of a Wyner-Ziv coder followed by a Costa coder, performs best.
\end{abstract}

\section{INTRODUCTION}

This paper considers broadcasting correlated Gaussian sources and aims to characterize mean squared-error (MSE) distortion pairs that are simultaneously achievable at two receivers using hybrid digital-analog (HDA) coding schemes. It is known that the separate design of source and channel coding due to Shannon does not in general lead to the optimal performance theoretically attainable (OPTA) in networks. On the other hand, for the point-to-point transmission of a single Gaussian source through an additive white Gaussian noise (AWGN) channel it is well known that if the channel and source bandwidths are equal, simple uncoded transmission achieves OPTA. Uncoded (or analog) transmission in this case (and in the rest of this paper) means scaling the encoder input subject to the channel power constraint and transmitting it without explicit channel coding. In order to exploit the advantages of both analog transmission and digital techniques, various HDA schemes have been introduced in the literature, see e.g., [1], [3]-[9]. Broadcasting a single memoryless Gaussian source under bandwidth mismatch using HDA schemes is considered in [5], [8]. Bross et al. [10] show that there exists a continuum of HDA schemes with optimal performance for the transmission of a Gaussian source over an average-power-limited Gaussian channel with matched bandwidth. Tian and Shamai [11] generalize this result to the mismatched bandwidth case. Broadcasting a Gaussian source with memory is analyzed in [9].

*This work was supported in part by a Postdoctoral Fellowship from the Ontario Ministry of Research and Innovation (MRI) and by the Natural Sciences and Engineering Research Council (NSERC) of Canada.

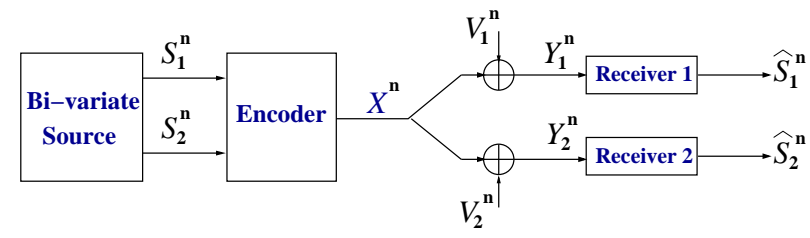

Fig. 1. Broadcasting a bivariate Gaussian source over a two-user powerlimited Gaussian broadcast channel.

Our system model is illustrated in Fig. 1. We aim to determine achievable distortion regions using HDA schemes for two cases; 1) the source bandwidth equals the channel bandwidth, 2) broadcasting with bandwidth compression. To our knowledge, apart from [12] in which Bross et al. analyzed uncoded transmission for broadcasting correlated Gaussian sources, no explicit distortion-regions have been established in the literature for broadcasting correlated Gaussian sources. We are also not aware of any prior work on HDA schemes for broadcasting correlated Gaussians either when the source and channel bandwidths are equal or when there is a bandwidth mismatch. Note that the source-channel separation theorem does not hold in broadcasting correlated sources.

\section{Problem Statement}

We consider broadcasting a bivariate Gaussian source across a two-user power-limited Gaussian broadcast channel. User $i(i=1,2)$ receives the transmitted signal corrupted by Gaussian noise with power $\sigma_{i}^{2}$ and wants to estimate the $i$ th component of the source. We assume $\sigma_{1}^{2}>\sigma_{2}^{2}$ and call user 1 the weak user and user 2 the strong user. Let $S_{1}$ and $S_{2}$ be correlated Gaussian random variables and let $\left\{\left(S_{1}(t), S_{2}(t)\right)\right\}_{t=1}^{\infty}$ be a stationary Gaussian memoryless vector source with marginal distribution that of $\left(S_{1}, S_{2}\right)$. We assume that $S_{1}(t)$ and $S_{2}(t)$ have zero mean and variance $\sigma_{S_{1}}^{2}$ and $\sigma_{S_{2}}^{2}$, respectively, and correlation coefficient $\rho \in$ $(-1,1)$.

We represent the first $n$ source samples by the data sequences $S_{1}^{n}=\left\{S_{1}(1), S_{1}(2), \cdots, S_{1}(n)\right\}$ and $S_{2}^{n}=$ $\left\{S_{2}(1), S_{2}(2), \cdots, S_{2}(n)\right\}$, respectively. The system is shown in Fig. 1. The source sequences $S_{1}^{n}$ and $S_{2}^{n}$ are jointly encoded to $X^{n}=\varphi\left(S_{1}^{n}, S_{2}^{n}\right)$, where the encoder function is of the form $\varphi: \mathbb{R}^{n} \times \mathbb{R}^{n} \rightarrow \mathbb{R}^{n}$. The transmitted sequence $X^{n}$ is average-power limited to $P>0$, i.e.,

$$
\frac{1}{n} \sum_{t=1}^{n} E\left[|X(t)|^{2}\right] \leq P .
$$

User $i$ observes the transmitted signal $X(t)$ corrupted by Gaussian noise $V_{i}(t)$ with power $\sigma_{i}^{2}$ so that each observation time $t=1,2,3, \ldots$ receiver $i$ observes

$$
Y_{i}(t)=X(t)+V_{i}(t), \quad i=1,2
$$


where the $V_{i}(t) \sim \mathcal{N}\left(0, \sigma_{i}^{2}\right)$ are independently distributed over $i$ and $t$, and are independent of the $X(t)$. Based on its channel output $Y_{i}^{n}$, user $i$ provides an estimate $\widehat{S}_{i}{ }^{n}=$ $\psi_{i}\left(Y_{i}^{n}\right)$, where $\psi_{i}: \mathbb{R}^{n} \rightarrow \mathbb{R}^{n}$ is a decoding function. The quality of the estimate is measured by the average MSE distortion $\Delta_{i}=\frac{1}{n} E\left[\sum_{t=1}^{n}\left|S_{i}(t)-\widehat{S}_{i}(t)\right|^{2}\right]$. Let $\mathcal{F}^{(n)}(P)$ denote all encoder and decoder functions $\left(\varphi, \psi_{1}, \psi_{2}\right)$ defined as above. For a particular coding scheme $\left(\varphi, \psi_{1}, \psi_{2}\right)$, the performance is determined by the channel power constraint $P$ and the incurred distortions $\Delta_{1}$ and $\Delta_{2}$ at the receivers. For any given power constraint $P>0$, the distortion region $\mathcal{D}$ is defined as the convex closure of the set of all distortion pairs $\left(D_{1}, D_{2}\right)$ for which $\left(P, D_{1}, D_{2}\right)$ is achievable, where a power-distortion pair $\left(P, D_{1}, D_{2}\right)$ is achievable if for any $\delta>0$, there exists $n_{0}(\delta)$ such that for any $n \geq n_{0}(\delta)$ there exists $\left(\varphi, \psi_{1}, \psi_{2}\right) \in \mathcal{F}^{(n)}(P)$ with distortions $\Delta_{i} \leq D_{i}+\delta$ $(i=1,2)$.

\section{Distortion Regions with Matched Bandwidth}

\section{A. Uncoded Transmission}

In [12] for the above problem an achievable distortion region is obtained based on analyzing the uncoded transmission in broadcasting a bivariate Gaussian source. In this approach, a linear combination of both components of a bivariate Gaussian source is transmitted across a powerlimited Gaussian broadcast channel. The transmitted signal can be expressed as

$$
X_{a}(t)=\tilde{\alpha} \sum_{i=1}^{2} a_{i} S_{i}(t)
$$

where $\tilde{\alpha}=\sqrt{\frac{P}{\operatorname{Var}\left(\sum_{i=1}^{2} a_{i} S_{i}(t)\right)}}, a_{i} \geq 0$ and $\operatorname{Var}\left(\sum_{i=1}^{2} a_{i} S_{i}(t)\right)=$ $a_{1}^{2} \sigma_{S_{1}}^{2}+a_{2}^{2} \sigma_{S_{2}}^{2}+2 a_{1} a_{2} \rho \sigma_{S_{1}} \sigma_{S_{2}}$. The scale factor $\tilde{\alpha}$ is chosen such that the channel power constraint is satisfied with equality. The received signal at receiver $i$ is then given by

$$
Y_{i}(t)=X_{a}(t)+V_{i}(t)=\tilde{\alpha} \sum_{i=1}^{2} a_{i} S_{i}(t)+V_{i}(t) .
$$

By evaluating the resulting MSE distortion, the set of simultaneously achievable distortion pairs at two users are as follows:

$$
D_{i}=\sigma_{S_{i}}^{2}-\frac{\tilde{\alpha}^{2}\left(a_{i} \sigma_{S_{i}}^{2}+a_{j} \rho \sigma_{S_{i}} \sigma_{S_{j}}\right)^{2}}{P+\sigma_{i}^{2}}, i, j=1,2, j \neq i
$$

It is shown in [12] that the uncoded scheme is optimal below a certain SNR-threshold.

\section{B. Joint Source-Channel Coding Schemes}

In our schemes, we will closely follow the notation and code constructions in [1]. Here we only give a high-level description and analyses of the schemes without detailed proofs. In particular, in many steps of the analysis we treat finite-blocklength coding schemes as idealized systems with asymptotically large blocklengths.

1) Layering with Analog and Costa Coding: This coding scheme has three layers and is similar to the scheme in [1] for broadcasting a single memoryless Gaussian source. The only difference between the two schemes is that we use a Wyner-Ziv encoder followed by a Costa encoder in the second layer, while the second layer of the scheme in [1] employs an HDA Costa coder (which will be explained in Section IV-A). Block diagrams of the encoder and the decoder are shown in Fig. 2. The first layer is the analog transmission layer. Here $X_{a}(t)=\alpha \sum_{i=1}^{2} a_{i} S_{i}(t)$, where $\alpha=$ $\sqrt{\frac{P_{a}}{\operatorname{Var}\left(\sum_{i=1}^{2} a_{i} S_{i}(t)\right)}}$. This layer is meant for both strong and weak users. Now fix $P_{1}$ and $P_{2}$ to satisfy $P=P_{a}+P_{1}+P_{2}$.

In the second layer, the first component of the source is first Wyner-Ziv coded at rate $R_{1}^{\prime}=\frac{1}{2} \log \left(1+\frac{P_{1}}{P_{2}+\sigma_{1}^{2}}\right)$ using an estimate of $S_{1}^{n}$ at the receiver as side information. The Wyner-Ziv index, $m_{1} \in\left\{1,2, \cdots, 2^{n R_{1}^{\prime}}\right\}$, is then encoded using Costa's "dirty paper" coding treating the analog transmission layer, $X_{a}^{n}$, as an interference. Let $U_{1}$ be an auxiliary random variable given by $U_{1}=X_{1}+\alpha_{1} X_{a}$, where $X_{1} \sim \mathcal{N}\left(0, P_{1}\right)$ is independent of $X_{a} \sim \mathcal{N}\left(0, P_{a}\right)$ and the scaling factor $\alpha_{1}$ is set to be $\frac{P_{1}}{P_{1}+P_{2}+\sigma_{1}^{2}}$. We generate a length $n$ i.i.d. Gaussian codebook $\mathcal{U}_{1}$ with $2^{n I\left(U_{1} ; Y_{1}\right)}$ codewords, where each component of the codeword is Gaussian with zero mean and variance $P_{1}+\alpha_{1}^{2} P_{a}$, and each codeword is then randomly placed into one of $2^{n R_{1}^{\prime}}$ bins. Let $i\left(U_{1}^{n}\right)$ be the index of the bin containing $U_{1}^{n}$. For a given $m_{1}$, we look for an $U_{1}^{n}$ such that $i\left(U_{1}^{n}\right)=m_{1}$ and $U_{1}^{n}$ and $X_{a}^{n}$ are jointly typical. Then, we transmit $X_{1}^{n}=U_{1}^{n}-\alpha_{1} X_{a}^{n}$, where $U_{1}^{n}$ is meant to be decoded by the weak user.

In the third layer, which is meant for the strong user, the second component of the source, $S_{2}^{n}$, is also Wyner $\mathrm{Ziv}$ coded at rate $R_{2}^{\prime}=\frac{1}{2} \log \left(1+\frac{P_{2}}{\sigma_{2}^{2}}\right)$ using the estimate of $S_{2}^{n}$ at the receiver as side information. The Wyner-Ziv index, $m_{2} \in\left\{1,2, \cdots, 2^{n R_{2}^{\prime}}\right\}$, is then encoded using digital Costa coding that treats both $X_{a}^{n}$ and $X_{1}^{n}$ as interference and uses power $P_{2}$. Let $U_{2}$ be an auxiliary random variable given by $U_{2}=X_{2}+\alpha_{2}\left(X_{a}+X_{1}\right)$, where $X_{2} \sim \mathcal{N}\left(0, P_{2}\right), X_{1}$ and $X_{a}$ are independent from each other and $\alpha_{2}=\frac{P_{2}}{P_{2}+\sigma_{2}^{2}}$. Here we also create a length $n$ i.i.d. Gaussian codebook $\mathcal{U}_{2}$ with $2^{n I\left(U_{2} ; Y_{2}\right)}$ codewords, where each component of the codeword is Gaussian with zero mean and variance $P_{2}+$ $\alpha_{2}^{2}\left(P_{a}+P_{1}\right)$ and (randomly) evenly distribute them over $2^{n R_{2}}$ bins. Let $i\left(U_{2}^{n}\right)$ be the index of the bin containing $U_{2}^{n}$. For a given $m_{2}$, we look for an $U_{2}^{n}$ such that $i\left(U_{2}^{n}\right)=$ $m_{2}$ and $\left(U_{2}^{n}, X_{a}^{n}, X_{1}^{n}\right)$ are jointly typical. Then, we transmit $X_{2}^{n}=U_{2}^{n}-\alpha_{2}\left(X_{a}^{n}+X_{1}^{n}\right)$. As shown in Fig. 2.(a), we merge all three layers and transmit $X^{n}=X_{a}^{n}+X_{1}^{n}+X_{2}^{n}$.

An achievable distortion-region can be obtained by varying $P_{a}, P_{1}$ and $P_{2}$ subject to $P=P_{a}+P_{1}+P_{2}$. For a given $P_{a}, P_{1}$ and $P_{2}$, the achievable distortion pairs can be computed as follows. At the receiver (Fig. 2.(b)), an estimate of the first component of the source, $S_{1}^{n}$, is first obtained from the analog layer. This estimate acts as side information that can be used in refining the estimate of $S_{1}^{n}$ for the weak user using the $R_{1}^{\prime}$ decoded Wyner-Ziv bits (obtained by the Costa decoder of the second layer). Since $R_{1}^{\prime}$ equals the capacity of the channel with known interference at the encoder only, $I\left(U_{1} ; Y_{1}\right)-I\left(U_{1} ; X_{a}\right)=\frac{1}{2} \log \left(1+\frac{P_{1}}{P_{2}+\sigma_{1}^{2}}\right)$, the distortion in estimating $S_{1}^{n}$ at the weak user is given by the Wyner-Ziv distortion-rate function, $D_{1}^{*} 2^{-2 R_{1}^{\prime}}$, where $D_{1}^{*}=E\left[\left(S_{1}-E\left[S_{1} \mid Y_{1}\right]\right)^{2}\right]$ is the (idealized) MMSE from 


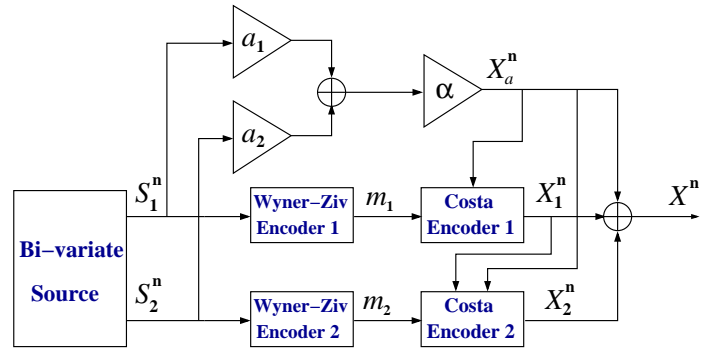

(a) Encoder

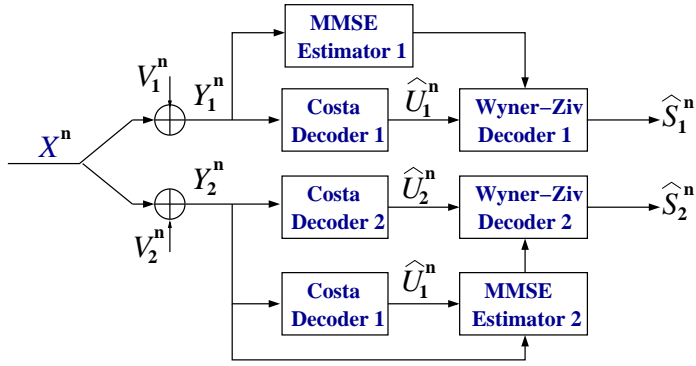

(b) Decoder

Fig. 2. Broadcasting a bivariate source $\left(S_{1}^{n}, S_{2}^{n}\right)$ by adopting the layering scheme with analog and Costa coding layers in [1].

the received $Y_{1}^{n}$. So the overall distortion seen at the weak user can be expressed as $D_{1}=D_{1}^{*}\left(1+\frac{P_{1}}{P_{2}+\sigma_{1}^{2}}\right)^{-1}$, where

$$
D_{1}^{*}=\sigma_{S_{1}}^{2}-\frac{\alpha^{2}\left(a_{1} \sigma_{S_{1}}^{2}+a_{2} \rho \sigma_{S_{1}} \sigma_{S_{2}}\right)^{2}}{P_{a}+P_{1}+P_{2}+\sigma_{1}^{2}} .
$$

Then, an estimate of $S_{2}^{n}$ can be determined from the first and the second layers. This estimate acts as side information for estimating $S_{2}^{n}$ (for the strong user) from the $R_{2}^{\prime}$ decoded Wyner-Ziv bits. Here, again, $R_{2}^{\prime}$ equals the capacity of the channel with known interference, $X_{a}^{n}$ and $X_{1}^{n}$, at the encoder only, i.e., $R_{2}^{\prime}=I\left(U_{2} ; Y_{2}\right)-I\left(U_{2} ; X_{a}, X_{1}\right)=\frac{1}{2} \log \left(1+\frac{P_{2}}{\sigma_{2}^{2}}\right)$. Thus, the distortion in estimating $S_{2}^{n}$ at the strong user is given by the Wyner-Ziv distortion-rate function, $D_{2}^{*} 2^{-2 R_{2}^{\prime}}$, where $D_{2}^{*}$ is the MMSE from the received $Y_{2}^{n}$ and the decoded $U_{1}^{n}$. So the overall distortion for the strong user is given by $D_{2}=D_{2}^{*}\left(1+\frac{P_{2}}{\sigma_{2}^{2}}\right)^{-1}$, where $D_{2}^{*}=\sigma_{S_{2}}^{2}-$ $\Gamma_{2}^{T} \Upsilon_{2}^{-1} \Gamma_{2}$,

$$
\Gamma_{2}=\left[\begin{array}{c}
\alpha\left(a_{2} \sigma_{S_{2}}^{2}+a_{1} \rho \sigma_{S_{1}} \sigma_{S_{2}}\right) \\
\alpha_{1} \alpha\left(a_{2} \sigma_{S_{2}}^{2}+a_{1} \rho \sigma_{S_{1}} \sigma_{S_{2}}\right)
\end{array}\right]
$$

and

$$
\Upsilon_{2}=\left[\begin{array}{cc}
P_{a}+P_{1}+P_{2}+\sigma_{2}^{2} & P_{1}+\alpha_{1} P_{a} \\
P_{1}+\alpha_{1} P_{a} & P_{1}+\alpha_{1}^{2} P_{a}
\end{array}\right] .
$$

2) Layering with Analog, Superposition and Costa Coding: This scheme also has three coding layers: analog, superposition, and Costa coding. In the second layer, we have two merged streams, similar to the case of broadcasting a single memoryless source over a broadcast channel [4], [13]. The first component of the source is broadcasted to two users. The first source encoder is an optimal Wyner-Ziv encoder with rate $R_{1}^{\prime \prime}=\frac{1}{2} \log \left(1+\frac{(1-\lambda) P_{1}}{\lambda P_{1}+P_{a}+P_{2}+\sigma_{1}^{2}}\right)$, and the second source encoder is an optimal Wyner-Ziv encoder for the residual error of the first encoder with rate $R_{2}^{\prime \prime}-R_{1}^{\prime \prime}=$ $\frac{1}{2} \log \left(1+\frac{\lambda P_{1}}{P_{a}+P_{2}+\sigma_{2}^{2}}\right)$. Then, we encode the Wyner-Ziv bits with capacity-achieving channel codes and transmit with

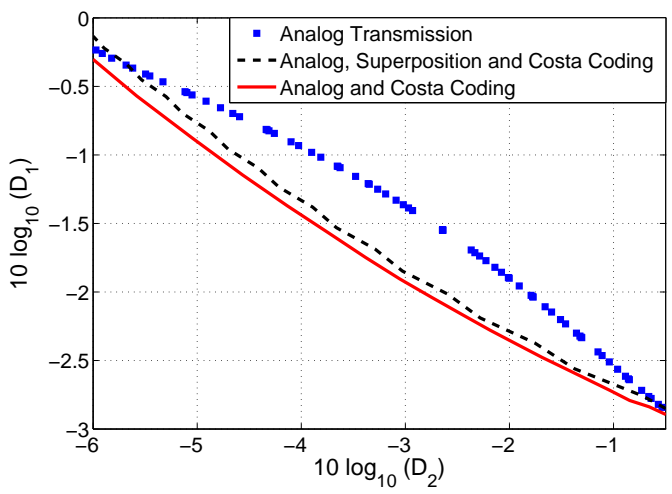

Fig. 3. Distortion regions in broadcasting a bivariate source with the correlation coefficient $\rho=0.2$.

powers $(1-\lambda) P_{1}$ and $\lambda P_{1}$, respectively. Since we require a rate of one channel use per source symbol, and the Gaussian source is successively refinable, by combining the Wyner-Ziv rate-distortion function with the pair of achievable rates for a broadcast channel $\left(R_{1}^{\prime \prime}, R_{2}^{\prime \prime}\right)$, the corresponding achievable distortion pairs are [4]: $D_{1}^{*} 2^{-2 R_{1}^{\prime \prime}}$ and $D_{1}^{*} 2^{-2 R_{2}^{\prime \prime}}$, where $D_{1}^{*}$ is given in (6). The coding scheme in the third layer is similar to that in the previous scheme.

The final distortion in estimating $S_{1}^{n}$ at the weak user is

$$
D_{1}=D_{1}^{*} 2^{-2 R_{1}^{\prime \prime}}=\frac{D_{1}^{*}}{1+\frac{(1-\lambda) P_{1}}{\lambda P_{1}+P_{a}+P_{2}+\sigma_{1}^{2}}} .
$$

At the strong user, first an estimate of the first component of the source can be obtained within distortion

$$
D_{12}^{*}=D_{1}^{*} 2^{-2 R_{2}^{\prime \prime}}=\frac{D_{1}^{*} 2^{-2 R_{1}^{\prime \prime}}}{1+\frac{\lambda P_{1}}{P_{a}+P_{2}+\sigma_{2}^{2}}}=\frac{D_{1}}{1+\frac{\lambda P_{1}}{P_{a}+P_{2}+\sigma_{2}^{2}}} .
$$

Then we obtain an estimate of $S_{2}^{n}$ from the above estimate of $S_{1}^{n}$ with the following distortion:

$$
D_{2}^{*}=\sigma_{S_{2}}^{2}\left(1-\rho^{2}\left(1-\frac{D_{12}^{*}}{\sigma_{S_{1}}^{2}}\right)\right) .
$$

This estimate of $S_{2}^{n}$ acts as side information in refining the estimate of $S_{2}^{n}$ (for the strong user) using the decoded Wyner-Ziv bits. The overall distortion for the strong user in estimating $S_{2}^{n}$ is thus given by $D_{2}=D_{2}^{*}\left(1+\frac{P_{2}}{\sigma_{2}^{2}}\right)^{-1}$.

3) Numerical Example: We transmit $n$ samples of a bivariate Gaussian source with the covariance matrix $\Lambda=$ $\left[\begin{array}{cc}1 & 0.2 \\ 0.2 & 1\end{array}\right]$ in $n$ uses of a power-limited broadcast channel to two users with observation noise variances $\sigma_{1}^{2}=-5 \mathrm{~dB}$ and $\sigma_{2}^{2}=0 \mathrm{~dB}$, respectively. The two-user broadcast channel has the power constraint $P=0 \mathrm{~dB}$. The boundaries of the distortion regions for the schemes presented in this section are shown in Fig. 3. We observe that the layering with analog transmission and Costa coding outperforms all other JSCC schemes, including analog transmission.

\section{Distortion Regions with BANDwidth COMPRESSION}

We next consider the problem of broadcasting a bivariate Gaussian source with 2:1 bandwidth compression. We want to transmit $k=2 n$ samples of a bivariate Gaussian source $\left(S_{1}^{k}, S_{2}^{k}\right)$ in $n$ uses of a power-limited broadcast channel to 


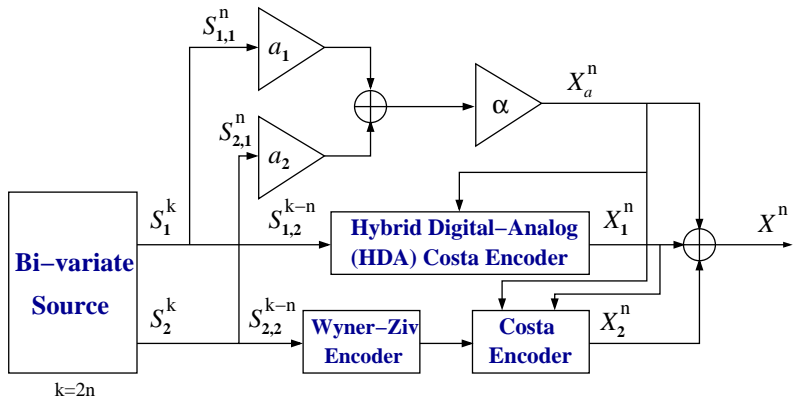

Fig. 4. Broadcasting a bivariate source $\left(S_{1}^{k}, S_{2}^{k}\right)$ with bandwidth compression using a three-layered coding provided in [1].

two users. The two-user broadcast channel has the power constraint $P$. We split both components of the bivariate Gaussian source into two equal length parts, i.e., we split $2 n$ samples of each source vector $S_{i}^{2 n}$ into two vectors of length $n: S_{i, 1}^{n}$ and $S_{i, 2}^{n}$.

\section{A. Layering with Analog, HDA Costa and Costa Coding}

This scheme is introduced in [1] for broadcasting a memoryless Gaussian source with bandwidth compression; see Fig. 4. In the first (analog) transmission layer, a linear combination of the first $n$ samples of the bivariate Gaussian source components are scaled such that the power of the transmitted signal in this layer $X_{a}^{n}$ becomes $P_{a}$. Here $X_{a}(t)=$ $\alpha \sum_{i=1}^{2} a_{i} S_{i, 1}(t)$ where $\alpha=\sqrt{\frac{P_{a}}{a_{1}^{2} \sigma_{S_{1}}^{2}+a_{2}^{2} \sigma_{S_{2}}^{2}+2 a_{1} a_{2} \rho \sigma_{S_{1}} \sigma_{S_{2}}}}$.

In the second and the third layers, we work on the remaining $n$ samples of the source components, i.e., $S_{1,2}^{n}$ and $S_{2,2}^{n}$, respectively. In the second layer, we apply the HDA Costa coding, presented in [1], to $S_{1,2}^{n}$ in order to produce $X_{1}^{n}$ with power $P_{1}$. Here, the source is not explicitly quantized and it appears in an analog form in the transmitted signal [1]. Let $U_{1}$ be an auxiliary random variable given by $U_{1}=X_{1}+\alpha_{1} X_{a}+K_{1} S_{1,2}$, where $X_{1} \sim \mathcal{N}\left(0, P_{1}\right)$, $X_{a} \sim \mathcal{N}\left(0, P_{a}\right)$, and $S_{1,2}$ are independent of each other, $\alpha_{1}=\frac{P_{1}}{P_{1}+P_{2}+\sigma_{1}^{2}}$, and $K_{1}^{2}=\frac{P_{1}^{2}}{\left(P_{1}+P_{2}+\sigma_{1}^{2}\right) \sigma_{S_{1}}^{2}}$. As in [1], we generate a random i.i.d. codebook $\mathcal{U}_{1}$ with $2^{n R_{1}}$ codewords, where each component of each codeword is Gaussian with zero mean and variance $P_{1}+\alpha_{1}^{2} P_{a}+K_{1}^{2} \sigma_{S_{1}}^{2}$ and $R_{1}=$ $\frac{1}{2} \log \left(\frac{P_{1}+\alpha_{1}^{2} P_{a}+K_{1}^{2} \sigma_{S_{1}}^{2}}{P_{1}}\right)$. For given $S_{1,2}^{n}$ and $X_{a}^{n}$, we find a $U_{1}^{n}$ such that $\left(U_{1}^{n}, S_{1,2}^{n}, X_{a}^{n}\right)$ is jointly typical and transmit $X_{1}^{n}=U_{1}^{n}-\alpha_{1} X_{a}^{n}-K_{1} S_{1,2}^{n}$.

In the third layer, $n$ samples of the second component of the source, $S_{2,2}^{n}$ are Wyner Ziv coded at rate $R_{2}^{\prime}=$ $\frac{1}{2} \log \left(1+\frac{P_{2}}{\sigma_{2}^{2}}\right)$ using the estimate of $S_{1,2}^{n}$ at the receiver as side information. The Wyner-Ziv index is then encoded using Costa coding that treats both $X_{a}^{n}$ and $X_{1}^{n}$ as interference and uses power $P_{2}=P-P_{a}-P_{1}$. The code construction as well as the encoding and decoding procedures are analogous to the ones described in Section III-B.1. Therefore, we transmit $X_{2}^{n}=U_{2}^{n}-\alpha_{2}\left(X_{a}^{n}+X_{1}^{n}\right)$. We merge all three layers and transmit $X^{n}=X_{a}^{n}+X_{1}^{n}+X_{2}^{n}$.

At the decoder, we look for an $U_{1}^{n}$ that is jointly typical with $Y_{1}^{n}$. The weak user estimates $S_{1}^{k}=\left(S_{1,1}^{n} S_{1,2}^{n}\right)$ by MMSE estimation from the received signal $Y_{1}^{n}$ and the decoded $U_{1}^{n}$. Thus, the overall distortion seen at the weak user is [1]:

$$
D_{1}=\frac{n}{k} D_{11}+\left(1-\frac{n}{k}\right) D_{12}=\frac{1}{2} D_{11}+\frac{1}{2} D_{12},
$$

where $D_{1 j}(j=1,2)$, the MMSE distortion in estimating $S_{1, j}^{n}$ from $Y_{1}^{n}$ and $U_{1}^{n}$, is given by

$$
D_{1 j}=\sigma_{S_{1}}^{2}-\Gamma_{1 j}^{T} \Upsilon_{1 H D A}^{-1} \Gamma_{1 j}
$$

where

$$
\Gamma_{11}=\left[\begin{array}{c}
\alpha\left(a_{1} \sigma_{S_{1}}^{2}+a_{2} \rho \sigma_{S_{1}} \sigma_{S_{2}}\right) \\
\alpha_{1} \alpha\left(a_{1} \sigma_{S_{1}}^{2}+a_{2} \rho \sigma_{S_{1}} \sigma_{S_{2}}\right)
\end{array}\right], \Gamma_{12}=\left[\begin{array}{c}
0 \\
K_{1} \sigma_{S_{1}}^{2}
\end{array}\right],
$$

and

$$
\Upsilon_{1 H D A}=\left[\begin{array}{cc}
P_{a}+P_{1}+P_{2}+\sigma_{1}^{2} & P_{1}+\alpha_{1} P_{a} \\
P_{1}+\alpha_{1} P_{a} & P_{1}+\alpha_{1}^{2} P_{a}+K_{1}^{2} \sigma_{S_{1}}^{2}
\end{array}\right] .
$$

Then, an estimate of $S_{2}^{k}$ is obtained from the first and the second layers. This estimate acts as side information for estimating $S_{2}$ (for the strong user) using the decoded Wyner$\mathrm{Ziv}$ bits. The strong user estimates the second component of the source $S_{2}^{k}=\left(S_{2,1}^{n} S_{2,2}^{n}\right)$ from $Y_{2}^{n}$, the decoded $U_{1}^{n}$ and $U_{2}^{n}$. Hence the overall distortion for the strong user is given by $D_{2}=\frac{1}{2} D_{21}+\frac{1}{2} D_{22}$, where $D_{2 j}(j=1,2)$, the distortion in estimating $S_{2, j}^{n}$, is determined via the WynerZiv distortion-rate function:

$$
D_{2 j}=\left(\sigma_{S_{2}}^{2}-\Gamma_{2 j}^{T} \Upsilon_{2 H D A}^{-1} \Gamma_{2 j}\right)\left(1+\frac{P_{2}}{\sigma_{2}^{2}}\right)^{1-j},
$$

where

$\Gamma_{21}=\left[\begin{array}{c}\alpha\left(a_{2} \sigma_{S_{2}}^{2}+a_{1} \rho \sigma_{S_{1}} \sigma_{S_{2}}\right) \\ \alpha_{1} \alpha\left(a_{2} \sigma_{S_{2}}^{2}+a_{1} \rho \sigma_{S_{1}} \sigma_{S_{2}}\right)\end{array}\right], \Gamma_{22}=\left[\begin{array}{c}0 \\ K_{1} \rho \sigma_{S_{1}} \sigma_{S_{2}}\end{array}\right]$,

and

$$
\Upsilon_{2 H D A}=\left[\begin{array}{cc}
P_{a}+P_{1}+P_{2}+\sigma_{2}^{2} & P_{1}+\alpha_{1} P_{a} \\
P_{1}+\alpha_{1} P_{a} & P_{1}+\alpha_{1}^{2} P_{a}+K_{1}^{2} \sigma_{S_{1}}^{2}
\end{array}\right] .
$$

\section{B. Layering with Analog and Costa Coding}

Here, we also use three coding layers and they are the same as the ones in Section IV-A, except for the second layer. In the second layer, the $n$ samples of the second half of the first component of the source, $S_{1,2}^{n}$, are quantized at rate $R_{1}^{\prime}=\frac{1}{2} \log \left(1+\frac{P_{1}}{P_{2}+\sigma_{1}^{2}}\right)$. The quantization index is then encoded using Costa coding that treats $X_{a}^{n}$ as interference and uses power $P_{1}$. Therefore, we transmit $X_{1}^{n}=U_{1}^{n}-$ $\alpha_{1} X_{a}^{n}$, where $\alpha_{1}=\frac{P_{1}}{P_{1}+P_{2}+\sigma_{1}^{2}}$. We merge all three layers and transmit $X^{n}=X_{a}^{n}+X_{1}^{n}+X_{2}^{n}$.

At the receiver, the weak user estimates $S_{1}^{2 n}=\left(S_{1,1}^{n} S_{1,2}^{n}\right)$ by MMSE estimation from the received signal $Y_{1}^{n}$ and the decoded $U_{1}^{n}$. Thus the overall distortion seen at the weak user is given by

$$
D_{1}=\frac{1}{2}\left(\sigma_{S_{1}}^{2}-\Gamma_{11}^{T} \Upsilon_{1}^{-1} \Gamma_{11}\right)+\frac{1}{2} \frac{\sigma_{S_{1}}^{2}}{1+\frac{P_{1}}{P_{2}+\sigma_{1}^{2}}},
$$

where $\Gamma_{11}$ is given in (11) and

$$
\Upsilon_{1}=\left[\begin{array}{cc}
P_{a}+P_{1}+P_{2}+\sigma_{1}^{2} & P_{1}+\alpha_{1} P_{a} \\
P_{1}+\alpha_{1} P_{a} & P_{1}+\alpha_{1}^{2} P_{a}
\end{array}\right] .
$$

The strong user estimates the second component of the 
source $S_{2}^{2 n}=\left(S_{2,1}^{n} S_{2,2}^{n}\right)$ within the overall distortion

$$
\begin{aligned}
D_{2}= & \frac{1}{2}\left(\sigma_{S_{2}}^{2}-\Gamma_{21}^{T} \Upsilon_{2}^{-1} \Gamma_{21}\right) \\
& +\frac{1}{2} \sigma_{S_{2}}^{2}\left(1-\rho^{2}\left(1-\frac{D_{12}^{*}}{\sigma_{S_{1}}^{2}}\right)\right)\left(1+\frac{P_{2}}{\sigma_{2}^{2}}\right)^{-1}(14)
\end{aligned}
$$

where $\Gamma_{21}$ is given in (12), $\Upsilon_{2}$ is provided in (7) and

$$
D_{12}^{*}=\frac{\sigma_{S_{1}}^{2}}{1+\frac{P_{1}}{P_{a}+P_{2}+\sigma_{2}^{2}}} .
$$

\section{Layering with Analog, Superposition and Costa Coding}

Analogously to the previous coding schemes, this scheme is three-layered with its layers identical to the ones presented in Section IV-A, except for the second layer. In the second layer, as in Section III-B.2, we use two merged streams. The second part of the first component of the source, $S_{1,2}^{n}$, is broadcasted to two users. The first source encoder is an optimal source encoder with rate $R_{1}^{\prime \prime}=$ $\frac{1}{2} \log \left(1+\frac{(1-\lambda) P_{1}}{\lambda P_{1}+P_{a}+P_{2}+\sigma_{1}^{2}}\right)$, and the second source encoder is an optimal encoder for the residual error of the first encoder with rate $R_{2}^{\prime \prime}-R_{1}^{\prime \prime}=\frac{1}{2} \log \left(1+\frac{\lambda P_{1}}{P_{a}+P_{2}+\sigma_{2}^{2}}\right)$. Then, we encode the quantization bits with capacity-achieving channel codes and transmit the resulting streams under powers $(1-\lambda) P_{1}$ and $\lambda P_{1}$, respectively.

The weak user forms an MMSE estimate of $S_{1}^{2 n}$ with the following distortion:

$$
\begin{aligned}
D_{1}= & \frac{1}{2}\left(\sigma_{S_{1}}^{2}-\frac{\alpha^{2}\left(a_{1} \sigma_{S_{1}}^{2}+a_{2} \rho \sigma_{S_{1}} \sigma_{S_{2}}\right)^{2}}{\lambda P_{1}+P_{a}+P_{2}+\sigma_{1}^{2}}\right) \\
& +\frac{1}{2} \frac{\sigma_{S_{1}}^{2}}{1+\frac{(1-\lambda) P_{1}}{\lambda P_{1}+P_{a}+P_{2}+\sigma_{1}^{2}}} .
\end{aligned}
$$

At the strong user, first an estimate of $S_{1,2}^{n}$ can be obtained within distortion

$$
D_{12}^{*}=\frac{1}{1+\frac{\lambda P_{1}}{P_{a}+P_{2}+\sigma_{2}^{2}}} \times \frac{\sigma_{S_{1}}^{2}}{1+\frac{(1-\lambda) P_{1}}{\lambda P_{1}+P_{a}+P_{2}+\sigma_{1}^{2}}} .
$$

This estimate acts as side information for obtaining the estimate of $S_{2,2}^{n}$ using the decoded Wyner-Ziv bits. The resulting distortion for the strong user is thus given by

$$
\begin{aligned}
D_{2}= & \frac{1}{2}\left(\sigma_{S_{2}}^{2}-\Gamma_{21}^{T} \Upsilon_{2}^{-1} \Gamma_{21}\right) \\
& +\frac{1}{2} \sigma_{S_{2}}^{2}\left(1-\rho^{2}\left(1-\frac{D_{12}^{*}}{\sigma_{S_{1}}^{2}}\right)\right)\left(1+\frac{P_{2}}{\sigma_{2}^{2}}\right)^{-1}(16)
\end{aligned}
$$

Finally, note that if we set $\rho=1$ and $\sigma_{S_{1}}^{2}=\sigma_{S_{2}}^{2}$, then the results of [1], [9], which currently appear to be the best known results for broadcasting a Gaussian source with bandwidth compression, are obtained.

\section{Numerical Results}

We transmit $k=2 n$ samples of a bivariate Gaussian source $\left(S_{1}^{k}, S_{2}^{k}\right)$ with the covariance matrix $\Lambda=\left[\begin{array}{ll}1 & \rho \\ \rho & 1\end{array}\right]$ in $n$ uses of a power-limited broadcast channel to two users with observation noise variances $\sigma_{1}^{2}=-5 \mathrm{~dB}$ and $\sigma_{2}^{2}=0 \mathrm{~dB}$, respectively. The distortion regions for the schemes presented in this section are shown in Fig. 5 for two different correlation coefficients, $\rho=0.2$ and $\rho=0.8$.
ISIT 2009, Seoul, Korea, June 28 - July 3, 2009

We observe that the layering with analog, superposition

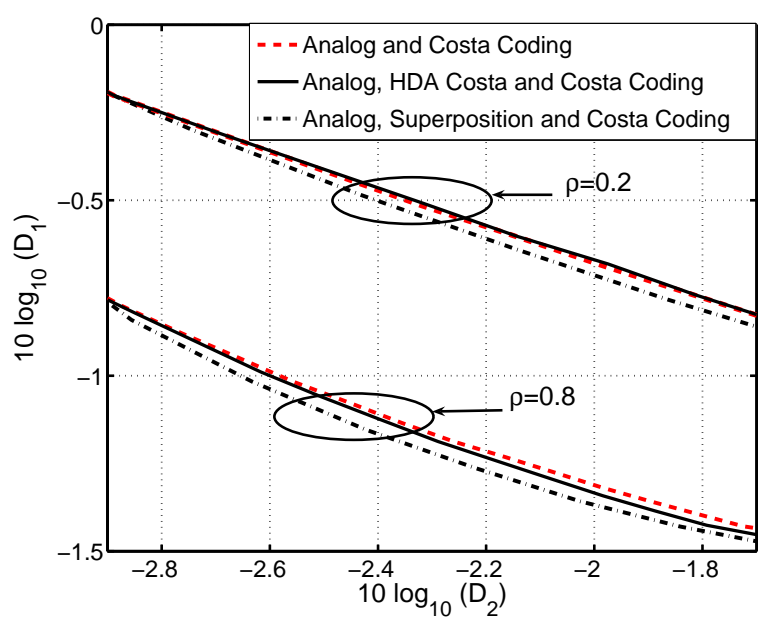

Fig. 5. Distortion regions of different HDA coding schemes. System parameters are $P=0 \mathrm{~dB}, \sigma_{1}^{2}=-5 \mathrm{~dB}$ and $\sigma_{2}^{2}=0 \mathrm{~dB}$.

and Costa coding of Section IV-C outperforms all other schemes in both cases. When the source components are highly correlated, layering with analog, HDA Costa, and Costa coding scheme performs better than the layering with analog and Costa coding scheme; however, the two two schemes perform similarly for small values of the correlation coefficient.

\section{REFERENCES}

[1] M. P. Wilson, K. Narayanan, and G. Caire, "Joint source channel coding with side information using hybrid digital analog codes," in Proc. IEEE Inf. Theory and Applications (ITA) Workshop, La Jolla, CA, Jan. 2007, pp. 299-308.

[2] V. M. Prabhakaran, R. Puri, and K. Ramchandran, "Colored Gaussian source-channel broadcast for heterogeneous (analog/digital) receivers," IEEE Trans. Inf. Theory, vol. 54, no. 4, pp. 1807-1814, Apr. 2008.

[3] S. Shamai, S. Verdu, and R. Zamir, "Systematic lossy source/channel coding," IEEE Trans. Inf. Theory, vol. 44, no. 2, pp. 564-579, Mar. 1998.

[4] B. Chen and G. W. Wornell, "Analog error-correcting codes based on chaotic dynamical systems," IEEE Trans. Commun., vol. 46, no. 7, pp. 881-890, Jul. 1998.

[5] U. Mittal and N. Phamdo, "Hybrid digital-analog (HDA) joint sourcechannel codes for broadcasting and robust communications," IEEE Trans. Inf. Theory, vol. 48, no. 5, pp. 1082-1102, May 2002.

[6] S. Sesia, G. Caire, and G. Vivier, "Lossy transmission over slowfading AWGN channels: a comparison of progressive, superposition and hybrid approaches," in Proc. IEEE ISIT, Adelaide, Australia, Sep. 2005.

[7] M. Skoglund, N. Phamdo, and F. Alajaji, "Hybrid digital-analog source-channel coding for bandwidth compression/expansion," IEEE Trans. Inf. Theory, vol. 52, no. 8, pp. 3757-3763, Aug. 2006.

[8] Z. Reznic, M. Feder, and R. Zamir, "Distortion bounds for broadcasting with bandwidth expansion," IEEE Trans. Inf. Theory, vol. 52, no. 8, pp. 3778-3788, Aug. 2006.

[9] V. M. Prabhakaran, R. Puri, and K. Ramachandran, "Hybrid analogdigital strategies for source-channel broadcast," in Proc. 43rd Allerton Conf. Commun., Contr., Comput., Allerton, IL, Sep. 2005.

[10] S. Bross, A. Lapidoth, and S. Tinguely, "Superimposed coded and uncoded transmissions of a Gaussian source over the Gaussian channel," in Proc. IEEE ISIT, Seattle, WA, Jul. 2006, pp. 2153-2155.

[11] C. Tian and S. Shamai, "A unified coding scheme for hybrid transmission of Gaussian source over Gaussian channel," in Proc. IEEE ISIT, Toronto, ON, Jul. 2008.

[12] S. Bross, A. Lapidoth, and S. Tinguely, "Broadcasting correlated Gaussians," in Proc. IEEE ISIT, Toronto, ON, Jul. 2008.

[13] M. C. Gastpar, "Separation theorems and partial orderings for sensor network problems," In Saligrama, Venkatesh (Ed.), Networked Sensing Information and Control, Springer, 2008. 